\title{
Pulse dynamics in SESAM-free electrically pumped VECSEL
}

\author{
Nikolai B. Chichkov, ${ }^{1,}$, (i) Amit Yadav, ${ }^{1}$ (i) Anton V. \\ Kovalev, ${ }^{2}$ (1) Semyon V. Smirnov, ${ }^{1}$ Markus Herper, ${ }^{3}$ Ksenia A. \\ FEdorova, ${ }^{4}$ (D) EVGENY A. VIKTOROV, ${ }^{2}$ AND EdIK U. RAFAILOV ${ }^{1}$ \\ ${ }^{1}$ Aston Institute of Photonic Technologies, Aston University, Birmingham B4 7ET, UK \\ ${ }^{2}$ ITMO University, Saint Petersburg, Russia \\ ${ }^{3}$ TRUMPF Photonic Components GmbH, Campus-Boulevard 79, 52074 Aachen, Germany \\ ${ }^{4}$ Faculty of Physics and Materials Sciences Center, Philipps-Universität Marburg, D-35032 Marburg, \\ Germany \\ *n.chichkov@aston.ac.uk
}

\begin{abstract}
Self-starting pulsed operation in an electrically pumped (EP) vertical-external-cavity surface-emitting-laser (VECSEL) without intracavity saturable absorber is demonstrated. A linear hemispherical cavity design, consisting of the EP-VECSEL chip and a 10\% output-coupler, is used to obtain picosecond output pulses with energies of $2.8 \mathrm{pJ}$ and pulse widths of $130 \mathrm{ps}$ at a repetition rate of $1.97 \mathrm{GHz}$. A complete experimental analysis of the generated output pulse train and of the transition from continuous-wave to pulsed operation is presented. Numerical simulations based on a delay-differential-equation (DDE) model of mode-locked semiconductor lasers are used to reproduce the pulse dynamics and identify different laser operation regimes. From this, the measured single pulse operation is attributed to FM-type mode-locking. The pulse formation is explained by strong amplitude-phase coupling and spectral filtering inside the EP-VECSEL.
\end{abstract}

Published by The Optical Society under the terms of the Creative Commons Attribution 4.0 License. Further distribution of this work must maintain attribution to the author(s) and the published article's title, journal citation, and DOI.

\section{Introduction}

Electrically pumped VECSELs are high-power, high-brightness semiconductor laser sources that benefit from low-cost, high-throughput production methods and ease of assembly and integration into optoelectronic devices [1-4]. They can be fabricated in 2D arrays with hundreds of emitters and combined with microlens arrays to realize high-power laser sources $[4,5]$. The use of an external cavity enables power scaling through the increase of the mode-field diameter, while simultaneously preserving diffraction limited beam profiles. As a consequence, EP-VECSELs provide orders-of-magnitude higher brightness values than vertical-cavity surface-emitting-laser (VCSEL) counterparts, with single-mode, continuous-wave (cw) output powers of up to $500 \mathrm{~mW}$ $[1,2]$.

Shortly after the development of high-power EP-VECSELs in 2003 [1], the first passively mode-locked EP-VECSEL was demonstrated [6]. Mode-locking was achieved with an intracavity semiconductor saturable absorber mirror (SESAM) and resulted in the generation of 56 ps pulses at a repetition rate of $1.1 \mathrm{GHz}$. This triggered further investigations into different laser designs and improvements of output pulse parameters during the last decade [7-10], motivated by the goal of developing compact, mass-producible ultrashort pulse laser systems. Passively mode-locked EP-VECSELs have been demonstrated with repetition rates in the range between $216 \mathrm{MHz}$ [9]

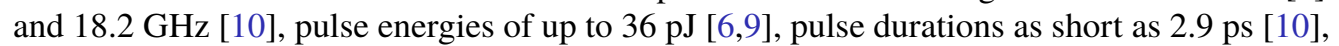
and peak powers of up to $4.7 \mathrm{~W}$ [10]. All of these EP-VECSELs have relied on SESAMs for 
mode-locking. The low pulse energies in EP-VECSELs require careful optimization of SESAM parameters and small beam diameters to achieve saturation [10].

EP-VECSELs exhibit a variety of different pulse dynamics, arising from the interplay between semiconductor nonlinearities (i.e. amplitude-phase coupling), transverse gain profiles, and external cavity modes. A range of theoretical models has been developed and applied for the analysis and optimization of pulse dynamics [11-13]. This research enabled the demonstration of new laser designs and mode-locking regimes. EP-VECSEL have been used to demonstrate the generation of temporal localized structures [14], vectorial solitons [15], and mode-locking by polarization gain-modulation [16]. One effect, however, that has so far only been observed in optically-pumped (OP) VECSELs is the so-called self-mode-locking, that is the observation of stable output pulse trains from OP-VECSELs without intracavity saturable absorber or external feedback [17-21]. The demonstrated output pulse characteristics closely resemble mode-locked operation, i.e. successive pulses are separated by the cavity round-trip time and the intensity drops to zero between pulses. As the term "self-mode-locking" suggests, this effect has not been fully understood so far, however, most publications used intracavity apertures and have attributed the mode-locking to Kerr-lensing inside the VECSEL chip.

Motivated by the self-mode-locking observed in OP-VECSELs, this paper investigates the pulse dynamics in an EP-VECSEL without SESAM and with no external feedback. Avoiding the use of a SESAM is not primarily done to achieve improvements in terms of laser design or cost. Instead, it is used to isolate and study the pulse dynamics which arise purely from the optical properties of the EP-VECSEL, with the goal to improve the understanding of laser operation and self-starting behaviour in mode-locked VECSELs.

Self-starting single pulse operation is demonstrated in the SESAM-free EP-VECSEL and a complete experimental characterization of the pulse dynamics and the transition from $\mathrm{cw}$ to pulsed operation is presented. The pulse dynamics and evolution of the laser regimes with increasing pump currents are reproduced by numerical simulations based on the delay-differential-equation (DDE) model described by Vladimirov et al. [11]. The single pulse operation is explained by FM-type mode-locking [22-29]. To the best of our knowledge, this is the first demonstration of self-starting pulsed operation in an EP-VECSEL without SESAM and with no external feedback.

\section{Experimental setup}

A schematic of the layer structure of the EP-VECSEL is shown in Fig. 1. Describing the layer structure bottom-up, the EP-VECSEL consists of a p-DBR with 32 mirror pairs, an oxide aperture with $100 \mu \mathrm{m}$ diameter for current confinement, three InGaAs quantum-wells (QW), an n-DBR with 11 mirror pairs and $82 \%$ reflectivity, and a GaAs current spreading layer. The current is injected through a disk-shaped bottom contact with a diameter of $40 \mu \mathrm{m}$ and a ring-shaped top-contact with an aperture of $200 \mu \mathrm{m}$. The size of the bottom contact was chosen to optimize the overlap between the VECSEL gain and the fundamental cavity mode [4]. The gain characteristics of EP-VECSELs with similar layer structures have been investigated by Pallmann et al. [7].

Figure 2 illustrates the laser and measurement setup. The EP-VECSEL was realized using a simple linear hemispherical cavity. The cavity was formed by the VECSEL chip on one end and a $10 \%$ output-coupler with a radius of curvature $(\mathrm{RoC})$ of $75 \mathrm{~mm}$ on the other end. The VECSEL was mounted on a copper heat-sink and temperature stabilized to $20^{\circ} \mathrm{C}$. The output-coupler was placed on a linear translation stage and the external cavity length was adjusted to approximately $76 \mathrm{~mm}$. The output beam was collimated with a $100 \mathrm{~mm}$ lens and the output power was measured directly after the collimating lens. The EP-VECSEL had a laser threshold current of $42 \mathrm{~mA}$ and a slope efficiency of $0.41 \mathrm{~mW} / \mathrm{mA}$. The maximum output power of $57 \mathrm{~mW}$ was obtained at a pump current of $183 \mathrm{~mA}$ and a voltage of $2.9 \mathrm{~V}$.

A polarizing Faraday isolator was used to prevent back-reflections from the characterization setup. Two waveplates, half-wave and quarter-wave, were used to maximize the transmission 


\section{OpticS EXPRESS}

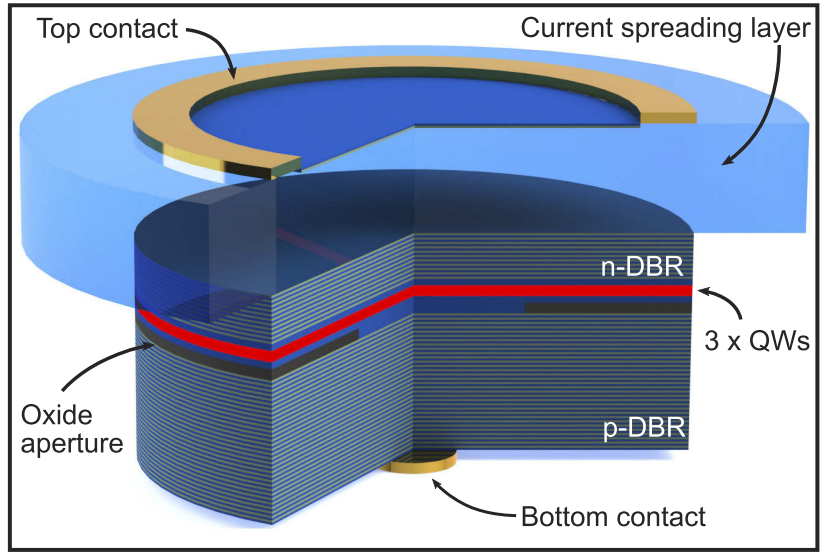

Fig. 1. Schematic of EP-VECSEL chip.

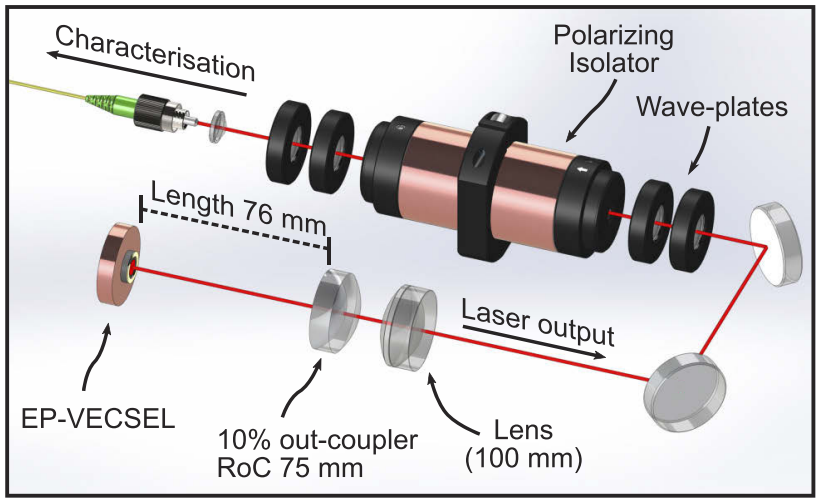

Fig. 2. SESAM-free EP-VECSEL setup.

through the isolator, accounting for all possible output polarizations. The transmitted beam was coupled into a single-mode fiber, with a cut-off wavelength of $780 \mathrm{~nm}$, which was connected to fiber-coupled measurement equipment. A second pair of waveplates was used to compensate for birefringence in the single-mode fiber and to adjust the input polarization of the fiber-coupled autocorrelater. To avoid back-reflections all optical elements were AR-coated and the fiber ends were polished at an $8^{\circ}$ angle (FC/APC connectors).

The generated output pulse trains were characterized with a $25 \mathrm{GHz}$ photodiode, connected to a $40 \mathrm{GHz}$ radio-frequency $(\mathrm{RF})$ spectrum analyzer and a $33 \mathrm{GHz}$ real-time oscilloscope. The photodiode and oscilloscope have a combined rise-time of 17 ps. A commercial autocorrelator (Femtochrome 103-XL) and optical spectrum analyzer were used to measure the temporal and spectral pulse profiles. All oscilloscope traces were recorded with a sampling rate of $80 \mathrm{GS} / \mathrm{s}$, and all optical spectra were measured with a resolution of $20 \mathrm{pm}$.

\section{Results}

\subsection{Pulsed operation}

Pulsed laser operation was achieved by careful alignment of the cavity length and output-coupler. The cavity length was adjusted to $76.0 \mathrm{~mm}$ and pulsed operation could be observed only for a narrow range of few hundred micrometers around that point. The measured oscilloscope traces 


\section{Optics EXPRESS}

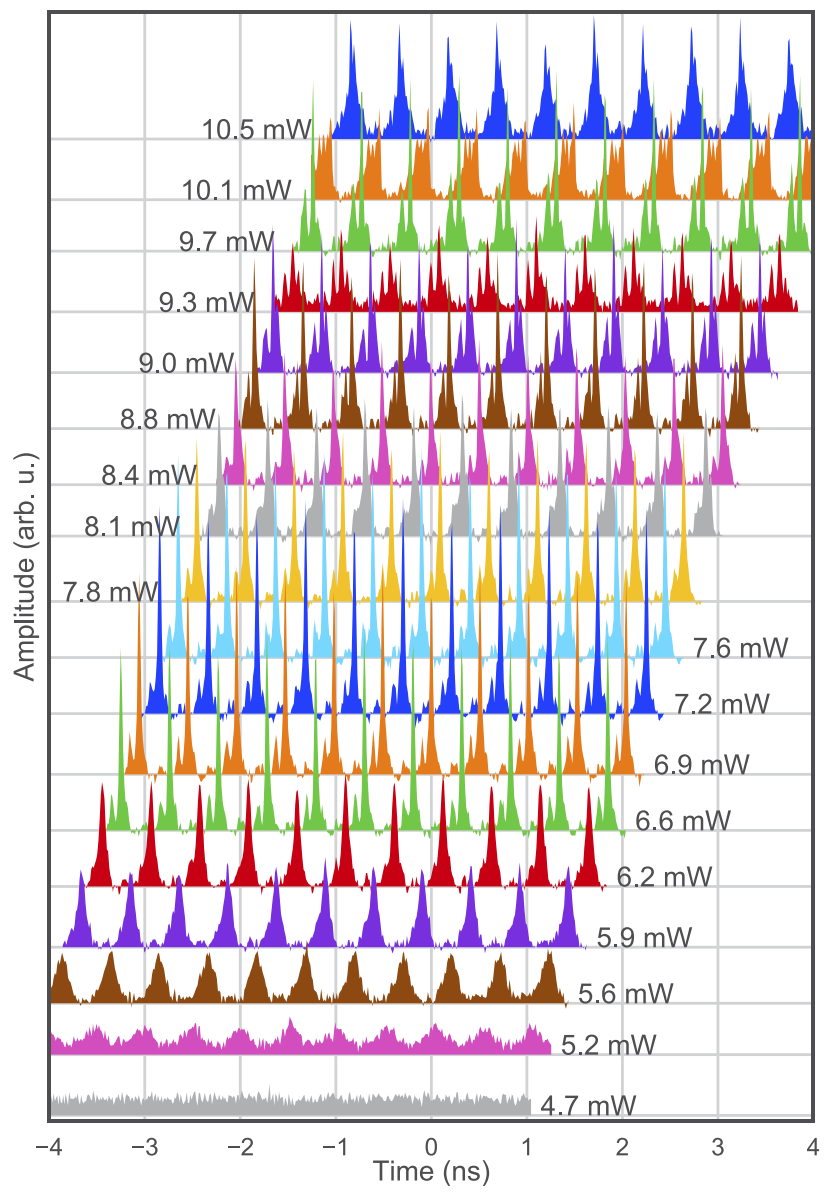

Fig. 3. Oscilloscope traces versus output power, plotted in steps of $1 \mathrm{~mA}$, starting at the pump current of $52.7 \mathrm{~mA}$ (corresponding to $4.7 \mathrm{~mW}$ ).

and optical spectra for different laser operation states are plotted in Fig. 3 and Fig. 4. The plots show the evolution of the pulse dynamics and laser spectrum for increasing values of the pump current. All measurements were recorded by a fully automated setup without any realignment or modifications for different laser currents.

Above the pump current threshold, the EP-VECSEL begins to operate in the cw regime at a wavelength of $983.09 \mathrm{~nm}$. The laser continues to operate in the $\mathrm{cw}$ regime for currents of up to $52.7 \mathrm{~mA}$. At this current, the laser produces an output power of $4.7 \mathrm{~mW}$ at a laser wavelength of $983.28 \mathrm{~nm}$. Further increase of the pump current to $53.7 \mathrm{~mA}$ leads to a sinusoidal modulation of the laser output.

Stable single pulse operation is obtained at a pump current of $54.6 \mathrm{~mA}$ with an output power of $5.6 \mathrm{~mW}$. The laser generates a continuous, uninterrupted output pulse train with pulse durations around 130 ps (see sections 3.2 and 3.3) and a repetition rate of $1.97 \mathrm{GHz}$. The onset of pulsed operation is accompanied by an abrupt increase in the laser bandwidth from $39 \mathrm{pm}$ to $61 \mathrm{pm}$ (full-width at half-maximum, FWHM). The single pulse operation is preserved when the pump current and output power are further increased to $56.6 \mathrm{~mA}$ and $6.2 \mathrm{~mW}$. The laser bandwidth increases to 105 pm and sub-100 ps pulse durations are obtained. However, the output pulse 


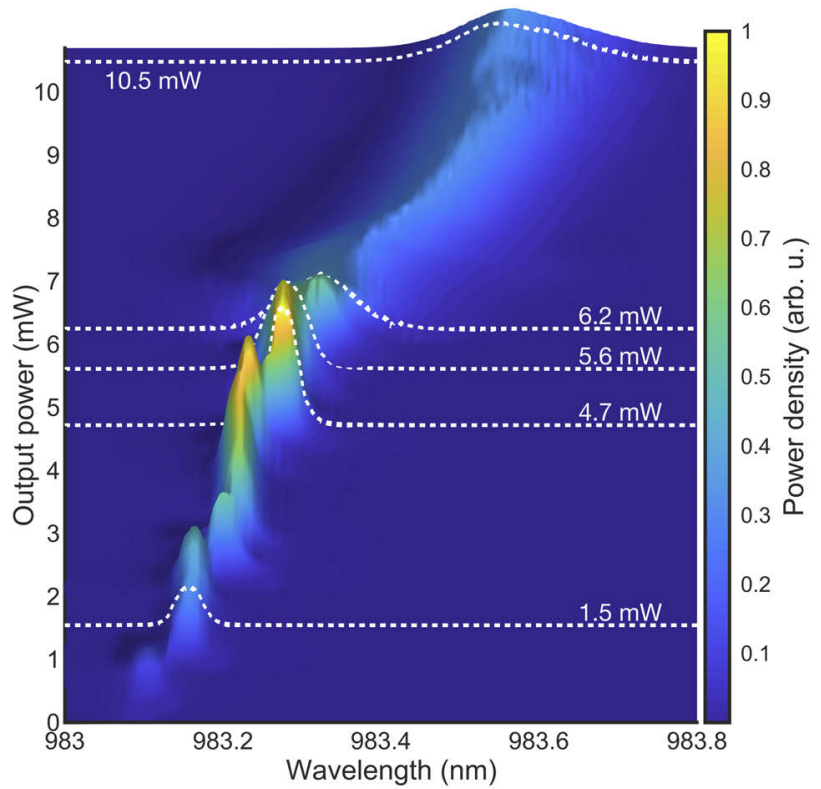

Fig. 4. Optical spectrum versus output power, plotted with a step size of $0.5 \mathrm{~mA}$.

trains become highly unstable with peak power, pulse duration, and energy fluctuations of 20-30 $\%$, occurring on time-scales of few $100 \mathrm{~ns}$.

Beyond the pump current of $56.6 \mathrm{~mA}$ (output power of $6.2 \mathrm{~mW}$ ), the output pulse trains become highly unstable and multi-pulsing, i.e. more than one pulse per round-trip, is observed. On time scales above $100 \mathrm{~ns}$, the oscilloscope traces show strong random fluctuations in pulse shape, pulse energy, and number of pulses. Separate pulses can still be distinguished in Fig. 3 but they break up or merge with other pulses over longer time scales. The laser switches to a random noise-like operation for output powers above $8.4 \mathrm{~mW}$. This operation mode is characterized by longer pulses with rapidly changing pulse shapes and an autocorrelation with a small sub-20 ps spike on top of a broad pedestal. The described changes in pulse dynamics are accompanied by a shift to longer wavelengths and continuous broadening of the optical spectrum. The laser wavelength shifts from $983.32 \mathrm{~nm}$ at an output power of $6.2 \mathrm{~mW}$ to $983.56 \mathrm{~nm}$ at $10.5 \mathrm{~mW}$. At the same time, the laser bandwidth increases from $105 \mathrm{pm}$ to $169 \mathrm{pm}$.

For higher output powers above $10.5 \mathrm{~mW}$ the output pulse trains begin to blur, i.e. the intensity does not drop to zero between pulses. At a pump current of $170.6 \mathrm{~mA}$ and output power of 53.7 $\mathrm{mW}$, the measured oscilloscope traces show a continuous signal with fast, random amplitude modulations.

The described changes in laser operation were reliably reproduced by successive measurements. Measurements for increasing and decreasing pump currents showed similar evolutions of pulse dynamics, optical spectra, and output powers. Turning the laser off and on again did not change the operation state. Finally, the pulsed operation was self-starting, in the sense that after blocking and unblocking the intracavity beam the laser directly returned to the same operation state.

\subsection{Single-pulse operation}

The remainder of this paper will focus on the single pulse operation observed for output powers between $5.6 \mathrm{~mW}$ and $6.2 \mathrm{~mW}$. A complete experimental characterization of the output pulses at $5.6 \mathrm{~mW}$ is presented in Fig. 5. The temporal pulse dynamics have been analyzed by use of a long-span oscilloscope trace, recorded over a time interval of $50 \mu$ s, which corresponds 


\section{Optics EXPRESS}

to 100,000 round trips. Slicing the oscilloscope trace into intervals corresponding to a single round-trip and plotting successive round-trips along a second axis results in the space-time diagram [15,30,31] shown in Fig. 5(a). The measured pulse durations (130 ps) are significantly longer than the rise-time of our measurement system (17 ps). As consequence, the oscilloscope traces provide a direct measure of the pulse shape and can be related to peak power and pulse energy (by normalizing the average amplitude to the output power). This approach allows to characterize the evolution of pulse parameters over multiple round-trips.
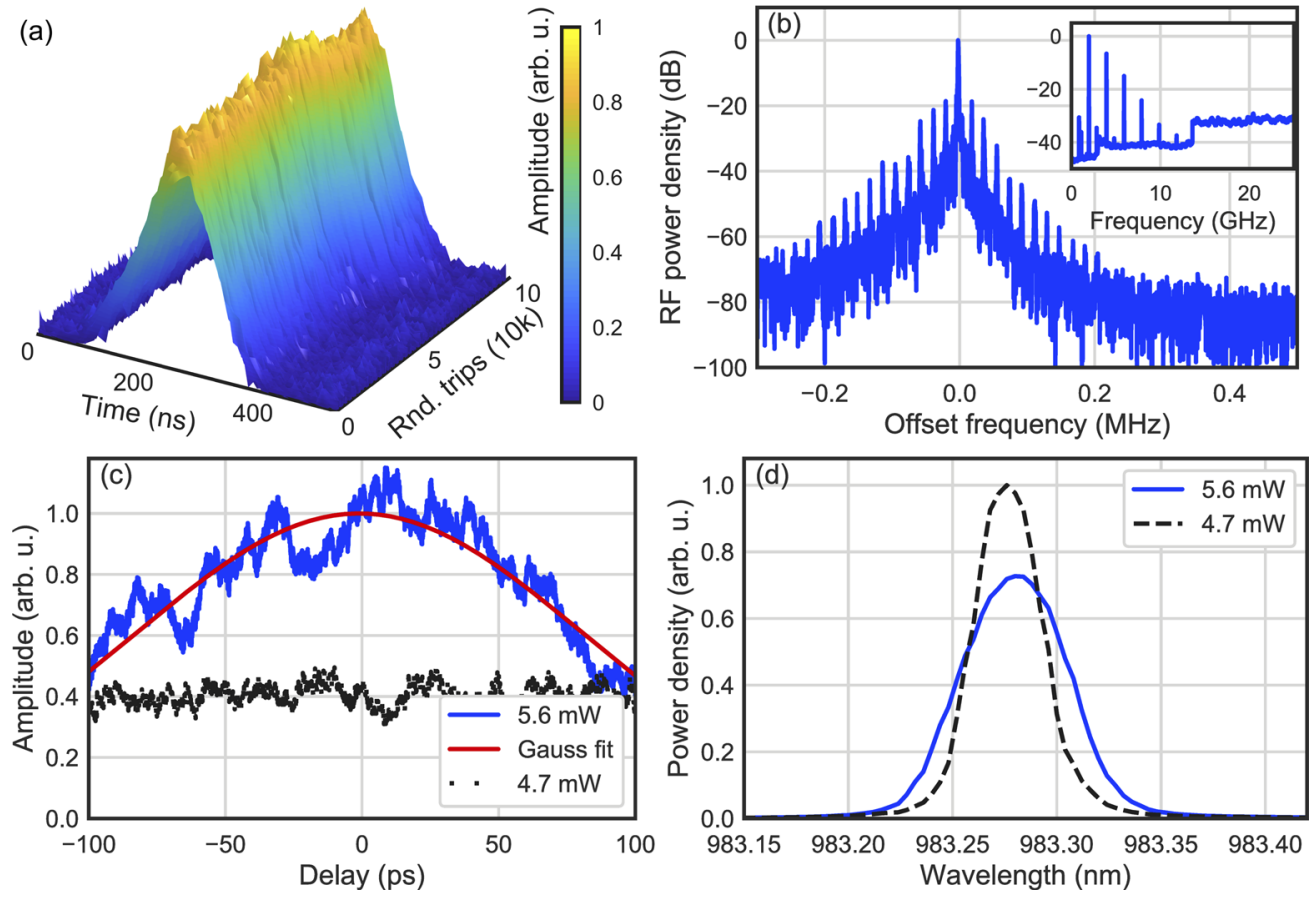

Fig. 5. Full characterisation of the single pulse operation at a pump current of $54.6 \mathrm{~mA}$ and with an output power of $5.6 \mathrm{~mW}$ : (a) Output pulse profile recorded on the oscilloscope over a time span of $50 \mu \mathrm{s}$, (b) Radio-frequency spectrum at the fundamental repetition rate and over the full measurement range of $25 \mathrm{GHz}$ (inset), (c) Intensity autocorrelation at 5.6 $\mathrm{mW}$ and directly before the onset of pulsing (dotted), (d) Optical spectrum at $5.6 \mathrm{~mW}$ and directly before the onset of pulsing (dashed).

The measured output pulse trains show fluctuations in peak amplitude, pulse duration, and energy (see Appendix A). The pulse shape, however, does not exhibit any significant changes over the complete measurement span. The output pulses have an average pulse width of $130.2 \mathrm{ps}$, which fluctuates with a standard deviation (SD) of $6.7 \mathrm{ps}$. The average pulse energy is $2.84 \mathrm{pJ}$ (0.09 $\mathrm{pJ} \mathrm{SD)}$ and the average peak power is $20.3 \mathrm{~mW}(0.8 \mathrm{~mW} \mathrm{SD})$.

The RF spectrum for the single pulse operation is plotted in Fig. 5(b). The RF peak at the fundamental frequency is located on top of a broad pedestal, starting at a level of approximately $-25 \mathrm{~dB}$. Additionally, the fundamental peak is surrounded by equally spaced side-peaks at levels of $-20 \mathrm{~dB}$ and spacing of approximately $20 \mathrm{kHz}$. The pedestal is in agreement with the observed pulse energy fluctuations, while the side peaks at $20 \mathrm{kHz}$ can be explained by periodic backward shifts of the pulse position (see Appendix A). The power densities of higher harmonics decrease exponentially with higher frequencies, dropping by $30 \mathrm{~dB}$ over $10 \mathrm{GHz}$. This decrease and the width of the RF spectrum are in agreement with the measured pulse shape and pulse durations of 130 ps. 


\section{Optics EXPRESS}

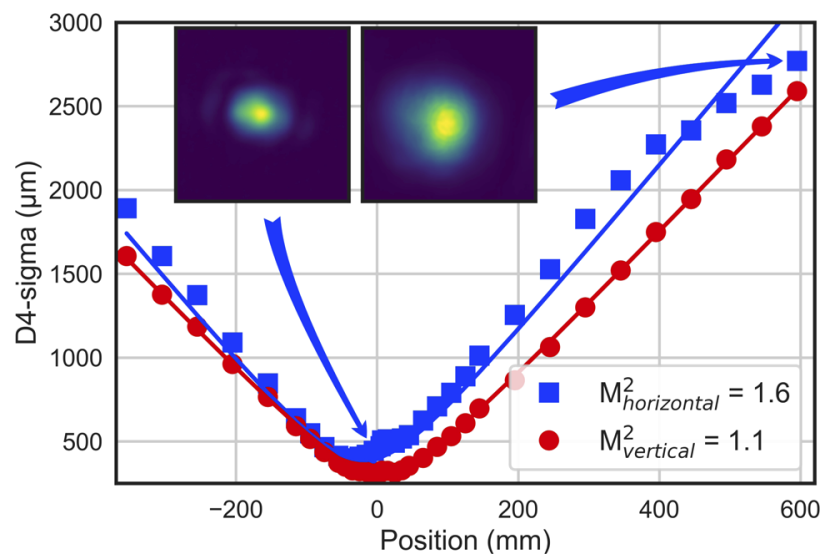

Fig. 6. Laser beam profile and $\mathrm{M}^{2}$-measurement for single pulse operation at $5.6 \mathrm{~mW}$.

Figure 5(c) shows the measured intensity autocorrelation (AC). A clear increase in the AC intensity is observed for the transition between $\mathrm{cw}$ and pulsed operation, consistent with the increase in peak power from $4.7 \mathrm{~mW}$ to $20.3 \mathrm{~mW}$. The AC trace is distorted by noise, due to the low peak powers of $20 \mathrm{~mW}$, and does not fit inside the $200 \mathrm{ps}$ window of the autocorrelator. Nonetheless, it shows the absence of sub-17 ps substructures, which would become visible as additional peaks in the center of the AC trace. From the Gaussian fit an AC width of $193 \mathrm{ps}$ and pulse width of $136 \mathrm{ps}$ are obtained, in good agreement with the oscilloscope measurements. Thus, the AC confirms that the temporal pulse profile is accurately resolved by the oscilloscope traces.

The optical spectrum of the EP-VECSEL in single pulse operation is plotted in Fig. 5(d). As already mentioned, the spectral width increases abruptly when the laser switches from $\mathrm{cw}$ to pulsed operation. The laser spectrum has a width of $61 \mathrm{pm}$ and corresponds to a Fourier-transform-limited (FTL) pulse duration of $15.2 \mathrm{ps}$.

Finally, in order to examine nonlinear lensing, the output beam profiles and evolution of the beam radius versus position were measured (see Fig. 6). In single pulse operation at $5.6 \mathrm{~mW}$, the EP-VECSEL produced a slightly multi-mode beam with $\mathrm{M}^{2}$ values of 1.6 and 1.1 in the horizontal and vertical directions. Comparison of the beam characteristics for $\mathrm{cw}$ and pulsed operation shows no significant changes. As the laser switches from $\mathrm{cw}$ to pulsed operation, the D4-sigma beam diameters at the beam waist change from $389.7 \mu \mathrm{m}$ to $390.5 \mu \mathrm{m}$ in the horizontal direction and from $321.4 \mu \mathrm{m}$ to $312.5 \mu \mathrm{m}$ in the vertical direction. These small changes in beam diameters suggest that nonlinear Kerr-lensing effects are negligible in the presented EP-VECSEL setup, which is in agreement with previously reported measurements of the nonlinear refractive index in GaAs-based VECSEL chips [32-35]. It was possible to achieve close to single-mode beam characteristics $\left(\mathrm{M}^{2}<1.1\right)$ by alignment of the output-coupler. Using this alignment, however, no stable single pulse operation could be obtained.

\subsection{Pulse chirp and temporal profile}

To obtain a better understanding of the single pulse regime it is necessary to determine the temporal phase of the output pulses. As already discussed, the presented oscilloscope traces provide a direct measure of the temporal pulse profile, since the $17 \mathrm{ps}$ rise-time of the measurement setup is significantly shorter than the pulse duration. Figure 7 shows the temporal pulse profile averaged over 100,000 round-trips. Having measured the temporal pulse profile and the optical spectrum, the iterative Gerchberg-Saxton (GS) algorithm can be applied to retrieve the temporal 


\section{Optics EXPRESS}

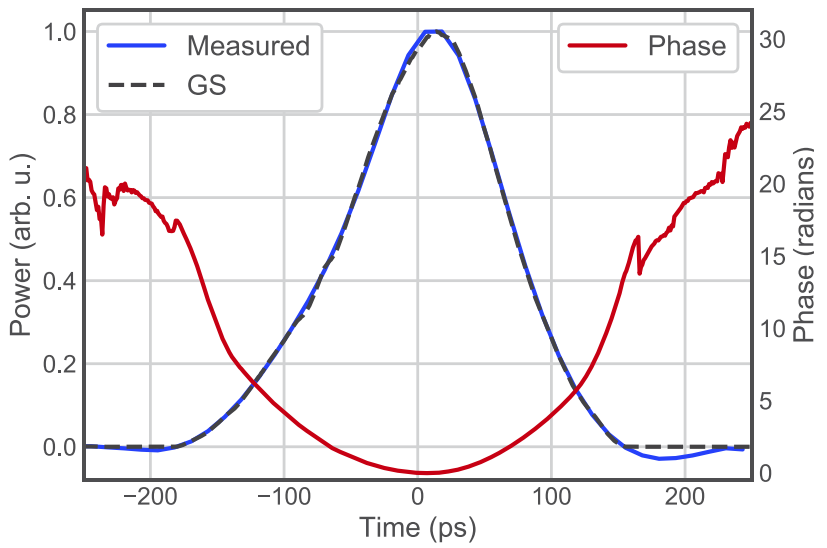

Fig. 7. Phase-retrieval using the GS algorithm: the average temporal pulse profile (blue) is compared to the temporal profile obtained from the GS algorithm (dashed) and the retrieved temporal phase (red).

phase [36,37]. The phase of a positively chirped Gaussian pulse with the same pulse duration and spectral width as the measured pulses is used as the initial guess for the algorithm.

The spectral phase retrieved with the GS algorithm is combined with the measured optical spectrum, giving a full description of the output pulses in the spectral domain. Fouriertransformation to the time-domain results in the temporal pulse profile and phase plotted in Fig. 7. From a third-order polynomial fit of the retrieved spectral phase a group-delay dispersion (GDD) of $1036 \mathrm{ps}^{2}$ is obtained. This pulse chirp results in output pulse durations which are almost one order of magnitude (factor 8.6) above the FTL duration. The corresponding time-bandwidth product (TBP) of 2.5 is unusual for mode-locked VECSELs, although comparable values of 1.3 have been measured for SESAM mode-locked devices with similar layer structure [7]. This TBP is closer to values obtained with mode-locked edge-emitters [38,39].

Unfortunately, the GS algorithm cannot be used to predict the sign of the GDD, due to the symmetry of the optical spectrum. The very large GDD value (corresponding to more than 50 $\mathrm{km}$ in fiber) also prevents the use of a standard grating compressor to stretch or compress the pulses and thereby determine the sign of the pulse chirp. Thus, the sign of the pulse chirp cannot be determined with the presented experimental setup. Nonetheless, the following theoretical discussion will show that the assumption of a positive linewidth-enhancement factor results in a positive pulse chirp, as is typically observed in passively mode-locked semiconductor lasers $[28,39]$.

\section{Discussion}

The principal experimental result is the demonstration of self-starting single pulse operation, explained in the following by FM-type mode-locking, in an EP-VECSEL without intracavity saturable absorber. Similar effects have already been observed in OP-VECSELs and attributed to Kerr-lensing inside the VECSELs. However, the intracavity peak powers in the EP-VECSEL were more than four orders of magnitude lower than the peak powers required for Kerr-lensing in OP-VECSELs [21,32,35]. Furthermore, no nonlinear lensing effects were observed in the beam profile and $\mathrm{M}^{2}$-measurements, which showed no significant difference between $\mathrm{cw}$ and pulsed operation. The possibility of mode-locking arising from polarization dependent feedback [16] has also been ruled out, by use of a Faraday isolator, AR-coatings, and angled fiber connectors, which ensured that no back-reflections or feedback were present in the experimental setup. 
The chirped pulsed dynamics and evolution of laser regimes with increasing pump currents can both be phenomenologically explained by strong amplitude-phase coupling inside the EPVECSEL. The strength of this coupling is conventionally characterized by the $\alpha$-factor [40]. No other assumptions of physical processes (e.g. Kerr lensing, spatial hole burning) are required. Following the approach introduced by Vladimirov et al. [11], the EP-VECSEL can be described by the following delay-differential-equation (DDE) model:

$$
\begin{aligned}
\gamma^{-1} \frac{d E(t)}{d t}+E(t) & =\sqrt{\kappa} e^{(1+i \alpha) G(t-T) / 2+i \varphi} E(t-T), \\
\gamma_{g}^{-1} \frac{d G(t)}{d t} & =g_{0}-G(t)-\left(e^{G(t)}-1\right)|E(t)|^{2},
\end{aligned}
$$

where $t$ is the time, $T$ is the cold cavity round trip time, $E(t)$ is the complex electric field envelope, $G(t)$ is the saturable gain, $\kappa$ accounts for the total non-resonant linear intensity losses per cavity round trip, $\gamma_{g}$ is the carrier density relaxation time, $g_{0}$ is the pump parameter, $\alpha$ is the linewidth-enhancement factor, and $\gamma$ is the gain bandwidth of the EP-VECSEL. As defined in [11], the parameter $\varphi=\Omega T$ accounts for the angular frequency detuning $\Omega$ between the modes of the external cavity and the gain filter which determines the $\mathrm{cw}$ frequency. The parameter values used for the following simulations are: $T=507 \mathrm{ps}, \gamma=789 \mathrm{GHz}, \gamma_{g}^{-1}=2.03 \mathrm{~ns}, \alpha=12$, $\kappa=0.65$.

The measured laser spectra exhibit a significant red shift with increasing pump currents (see Fig. 4). This shift is nearly linear with respect to the pump current in the vicinity of the lasing threshold and results from the shift of the EP-VECSEL gain to longer wavelengths and/or variation of the optical length of the laser cavity. In the DDE-model of Eq. (1), the red shift corresponds to decreasing values of the parameter $\varphi$ at higher pump currents. Thus, the red shift at increasing pump currents can be modelled by a linear relation between the pump parameter $g_{0}$ and $\varphi$. Using this approach, the numerical simulations predict a transition from $\mathrm{cw}$ operation to self-starting mode-locking to unstable pulsation, similar to the experimental results (see Fig. 3).

The results of the bifurcation analysis (see Appendix B for details) by means of numerical continuation [41] are shown in Fig. 8. The bifurcation diagram depicts the regions of possible operation modes, obtained by stability analysis of the solutions of Eq. (1) with the maximal gain mode at $\varphi=0$ as the initial condition. The stability analysis predicts four distinct dynamical regimes: $\mathrm{cw}$ operation close to the threshold, mode-locked operation, unstable pulsation, and mode-hopping. The dashed line in Fig. 8 indicates the modelled linear dependence of the $\varphi$ parameter and the pump parameter: $\varphi=a\left(g_{0} / g_{0, t h r}-1\right)$, with $a=-15 \pi$ and $g_{0, t h r}=0.108$. It approximately corresponds to the measured spectral red shift versus pump current in Fig. 4. Figure 8 shows transitions from $\mathrm{cw}$ operation to mode-locking and from mode-locking to unstable pulsing for increasing pump parameter values. This is in agreement with the experimental results presented in section 3.1.

Further bifurcation analysis was performed by direct numerical integration of Eq. (1) along the dashed line in Fig. 8, reproducing the increase of pump currents performed in the experiments. The simulation begins with the stationary solution at the lasing threshold. The pump parameter $g_{0}$ is increased step wise and Eq. (1) are integrated to find the stable solution after each step, using the solution of the previous step as starting condition. Figure 9 shows the extrema of the intensity oscillations as we progressively increase $g_{0}$. After a supercritical Hopf bifurcation at $g_{0}=1.92 \cdot g_{0, t h r}$, the amplitude of the oscillations rapidly increases to high values. This corresponds to the transition from $\mathrm{cw}$ to pulsed operation in the experiments. As we further increase $g_{0}$, the mode-locked operation becomes unstable via a secondary Hopf bifurcation, resulting in large amplitude chaotic pulsations. This is in agreement with the onset of pulse fluctuations and multi-pulsing observed in the experiments. Finally, the red shift evolution of the optical spectrum is shown in the inset of Fig. 9. The optical spectrum was calculated by the fast 


\section{OpticS EXPRESS}

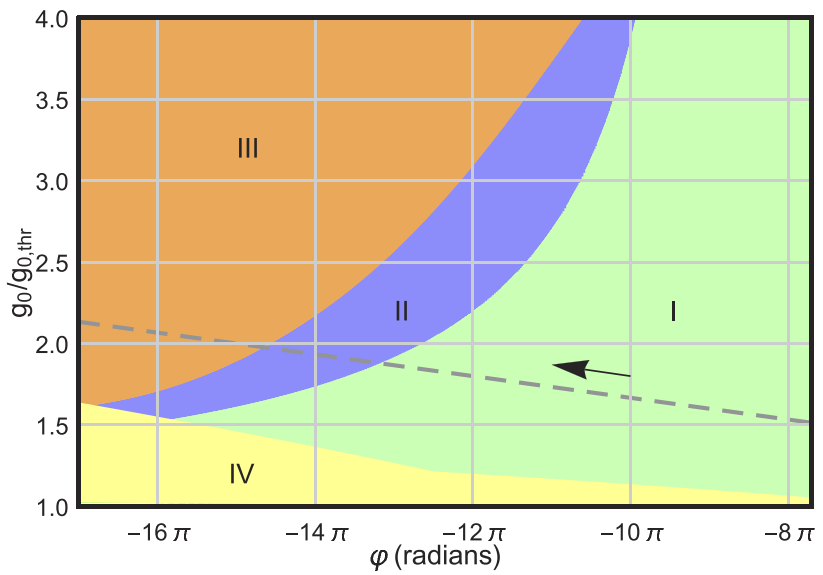

Fig. 8. Bifurcation diagram in the $\left(\varphi, g_{0}\right)$ plane showing the operation ranges of the following laser regimes: cw operation (I), mode-locking (II), unstable pulsation (III), and mode-hopping (IV). The dashed line shows the modelled linear relation between $\varphi$ and $g_{0}$.

Fourier transform of $E(t)$ and filtered with a Gaussian filter with a standard deviation of $6.2 \mathrm{GHz}$, corresponding to the $20 \mathrm{pm}$ resolution of the optical spectrum analyzer.

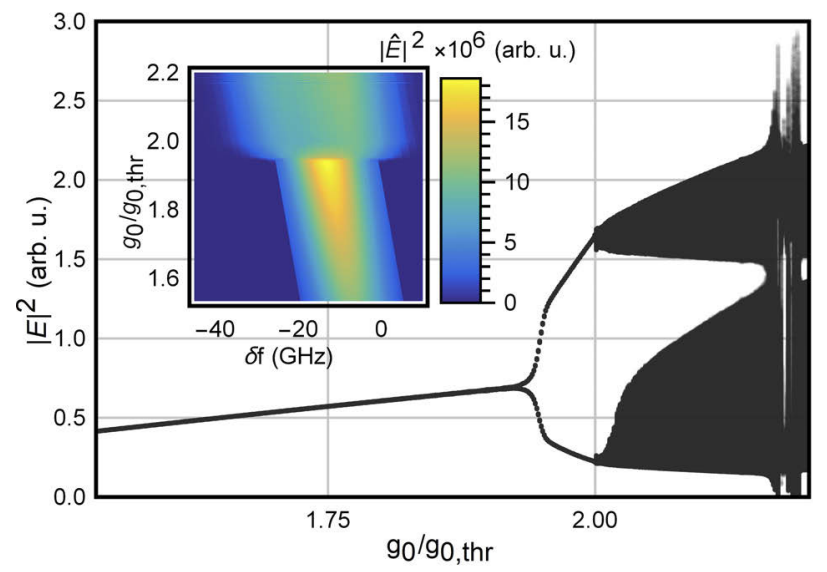

Fig. 9. Bifurcation diagram showing the laser intensity extrema of different dynamical regimes for increasing pump parameter values (along the dashed line in Fig. 8). Inset: evolution of the optical spectrum $\hat{E}(\delta f)$, where $\delta f$ is the offset from the gain maximum.

Stable mode-locked operation is obtained for the pump parameter value of $g_{0}=1.99 \cdot g_{0, t h r}$ and is similar to the single pulse operation described in section 3.2. The corresponding pulse train, temporal pulse profile, frequency modulation, and RF spectrum are plotted in Fig. 10. The pulses have a duration of $196 \mathrm{ps}$ and exhibit a linear positive chirp of $1223 \mathrm{ps}^{2}$, obtained by a third order polynomial fit of the spectral phase. These values are in good qualitative agreement with the experimentally obtained values of $136 \mathrm{ps}$ and $1036 \mathrm{ps}^{2}$. The strong pulse chirp leads to a modulation of the instantaneous frequency with a modulation amplitude of more than $10 \mathrm{GHz}$. The RF spectrum and the exponential decrease of the RF power densities of higher harmonics are in agreement with the measurements shown in Fig. 5(b). The main discrepancy between the numerical and experimental results is the cw background at a level of $15 \%$ in the simulated pulse train. The measured oscilloscope traces exhibit no cw background and were recorded with a 

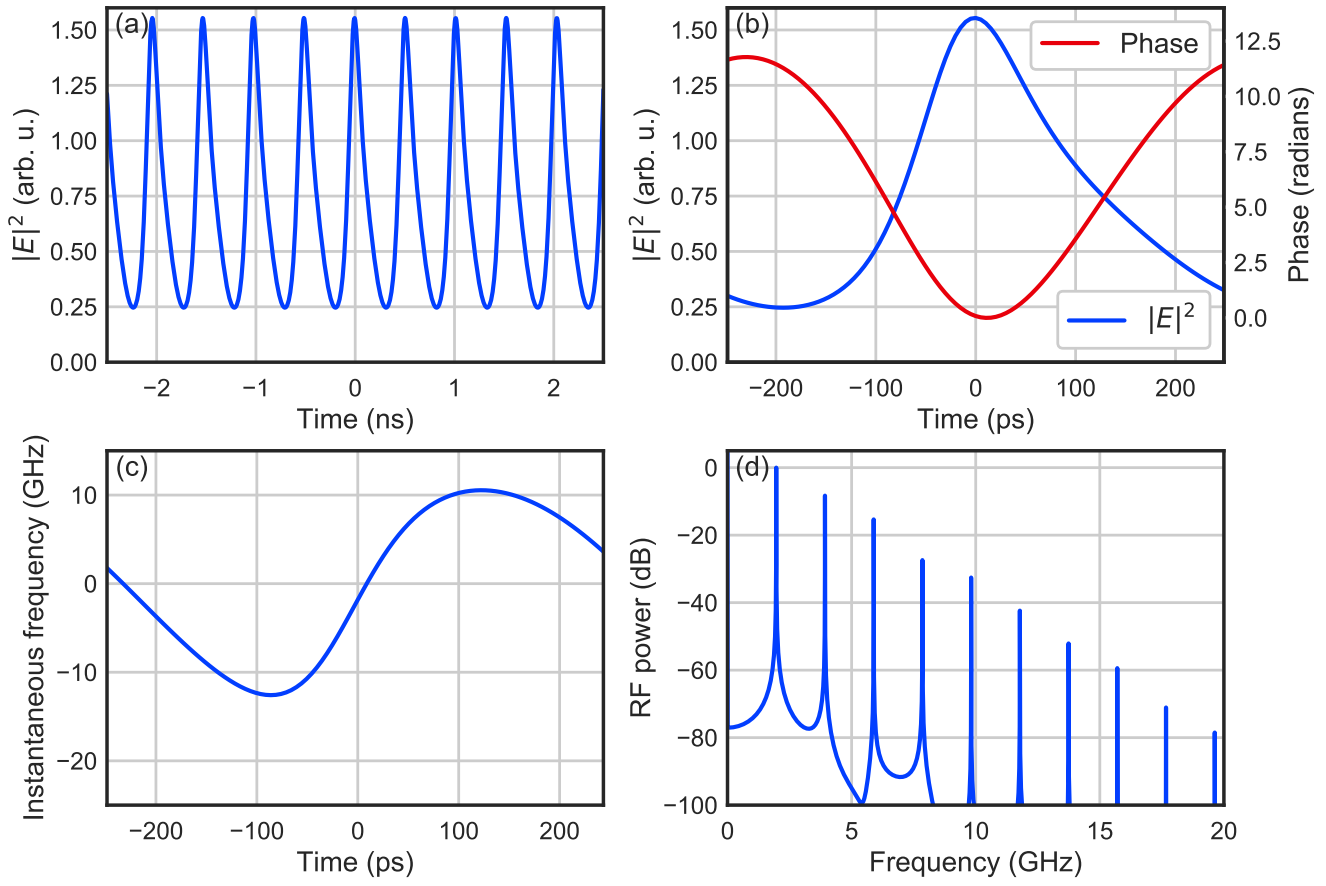

Fig. 10. Simulation of mode-locked operation at $\varphi=-14.92 \pi$ and $g_{0}=1.99 \cdot g_{0, t h r}$ : (a) pulse train, (b) temporal pulse intensity and phase variations, (c) instantaneous frequency, and (d) RF spectrum.

signal-to-noise ratio of 5\%. Better agreement with the experimental results (i.e. a smaller $\mathrm{cw}$ background) can be obtained by use of larger $\alpha$-factors in the simulations.

The numerical simulations demonstrate that the single pulse operation arises from the interplay of the spectral filtering and strong amplitude-phase coupling in the EP-VECSEL. The amplitude-phase coupling leads to frequency-modulations of the intracavity laser signal (see Fig. 10(c)), which are transformed to amplitude modulations by the gain filter. Thus, the mechanism responsible for the demonstrated single pulse operation can be classified as passive frequency-modulated (FM) mode-locking [42].

Passive FM-type mode-locking is well known in the literature [24,26-29] and has been conventionally associated with output fields possessing small intensity and large frequency modulations, which can be further transformed to pulses using dispersion compensation [29]. The primary effects responsible for FM-type mode-locking are usually spatial hole burning and four-wave mixing nonlinearities. This conventional FM-type mode-locking must be distinguished from the presented numerical results, which demonstrate a distinct type of FM mode-locking, caused by strong amplitude-phase modulations in the EP-VECSEL. This is accompanied by large amplitude modulations, which are comparable with amplitude-modulated (AM) mode-locking induced by saturable absorbers. Hence, the numerical results show passive FM-type mode-locking which produces FM and AM laser output.

A strong feedback signal at the external cavity round trip frequency causes fluctuations of the cavity mode frequency leading to phase and frequency modulations observed in the numerical simulations. In case of no detuning (i.e. $\Omega=0$ ) and small $\alpha$-factors, the FM efficiency is low and results only in a small amplitude damped modulation of the $\mathrm{cw}$ operation. The bifurcation 
analysis of Eq. (1) reveals that when the cavity modes become detuned against the gain filter (i.e. $\Omega \neq 0$ ), as consequence of the experimentally observed red shift of the optical spectrum, the perturbations of the electric field become uncoupled through a supercritical Hopf bifurcation. The experimentally observed transition from $\mathrm{cw}$ operation to near-sinusoidal oscillations of the laser output in Fig. 3 confirms this numerical result. The intensity oscillations become larger with increasing pump currents and stronger detuning. The combination of amplitude-phase coupling and spectral filtering produces periodic frequency oscillations and results in the generation of short large amplitude pulses with a small pedestal. The FM efficiency, i.e. AM depth and level of the pedestal, is determined by the $\alpha$-factor. Use of larger $\alpha$-factors increases the peak power and reduces the pedestal. While the mode-locked operation can also be obtained for smaller $\alpha$-factors, the experimentally observed pulse chirp and prominent pulses suggest a large $\alpha$-factor. The experimental results could be reproduced with an $\alpha$-factor of 12, which is within the range of values reported for VCSELs and VECSELs [43,44]. As the pump parameter and detuning increase, the pedestal decreases and the modulation becomes unstable, resulting in a slow amplitude modulation of the pulse train. Further increases in pump power eventually lead to satellite pulse fluctuations, again in good qualitative agreement with the experimental results.

In summary, the experimental results can be qualitatively explained by use of the simple DDE-model in Eq. (1) and the assumption of strong amplitude-phase coupling. The model accurately reproduces the observed transitions from $\mathrm{cw}$ operation to mode-locking and from mode-locking to unstable pulsation. Good agreement in terms of pulse durations and pulse chirp is obtained. Based on the numerical results, the single pulse operation of the EP-VECSEL is attributed to FM-type mode-locking and the pulse formation is explained by spectral filtering of the frequency-modulated electric field. Some experimental effects, however, could not be reproduced by the DDE-model. The measured periodic shifts in pulse position at $20 \mathrm{kHz}$, the peak power fluctuations, and the level of the $\mathrm{cw}$ background (i.e. pedestal) could not be reproduced. Additional experiments and the use of more sophisticated models, incorporating background noise, the microcavity of the EP-VECSEL [12,45], and third-order-dispersion [13] are required to further improve understanding of the pulse dynamics and the transformation of FM-type mode-locking to AM pulse trains.

\section{Conclusion}

In this paper, self-starting single pulse operation from a SESAM-free EP-VECSEL has been demonstrated and a complete experimental characterisation of the pulse dynamics and pulse parameters has been presented. The SESAM-free EP-VECSEL generated strongly chirped output pulses with durations of $130 \mathrm{ps}$ and pulse energies of $2.8 \mathrm{pJ}$. The presented results could be reproduced with other EP-VECSEL samples with identical layer structure.

A simple DDE model has been presented and used to explain the measured pulse dynamics, which have been attributed to FM-type mode-locking. Good qualitative agreement between numerical simulations and experimental results has been achieved in terms of pulse shape, chirp, and duration. Further research will have to focus on the accurate characterization of EP-VECSEL properties (specifically the $\alpha$-factor), the development of quantitative models for the prediction and improvement of pulse parameters, and the stabilization of pulse dynamics.

To the best of our knowledge, this is the first demonstration of self-starting pulsed operation in an EP-VECSEL without saturable absorber or external feedback. Additionally, this is the first theoretical investigation of FM-type mode-locking in a VECSEL and of its transformation to deep amplitude modulations of the laser output. These results have important implications for the design of mode-locked EP-VECSELs in the future. They demonstrate that a saturable absorber is not required to stimulate the formation of stable pulse trains from continuous-wave operation in EP-VECSELs (i.e. self-starting). Instead, the optical properties of EP-VECSELs 
can be exploited to trigger pulsed operation, while the absorber parameters can be optimized independently to improve mode-locking performance.

\section{Appendix A: Pulse fluctuations}

To analyze the fluctuations in pulse duration, energy, and peak power the following procedure was applied. First, the output pulse trains are recorded over 100,000 round trips (i.e. $50 \mu$ s) for the laser current of $54.6 \mathrm{~mA}$ and output power of $5.6 \mathrm{~mW}$. Then the oscilloscope trace is normalized so that the average amplitude corresponds to the measured output power. Afterwards, each round trip is analyzed separately to calculate the pulse energy (integration over one round-trip), FWHM of the pulse, and the peak power. The obtained values were used to calculate the histogram plotted in Fig. 11(left).
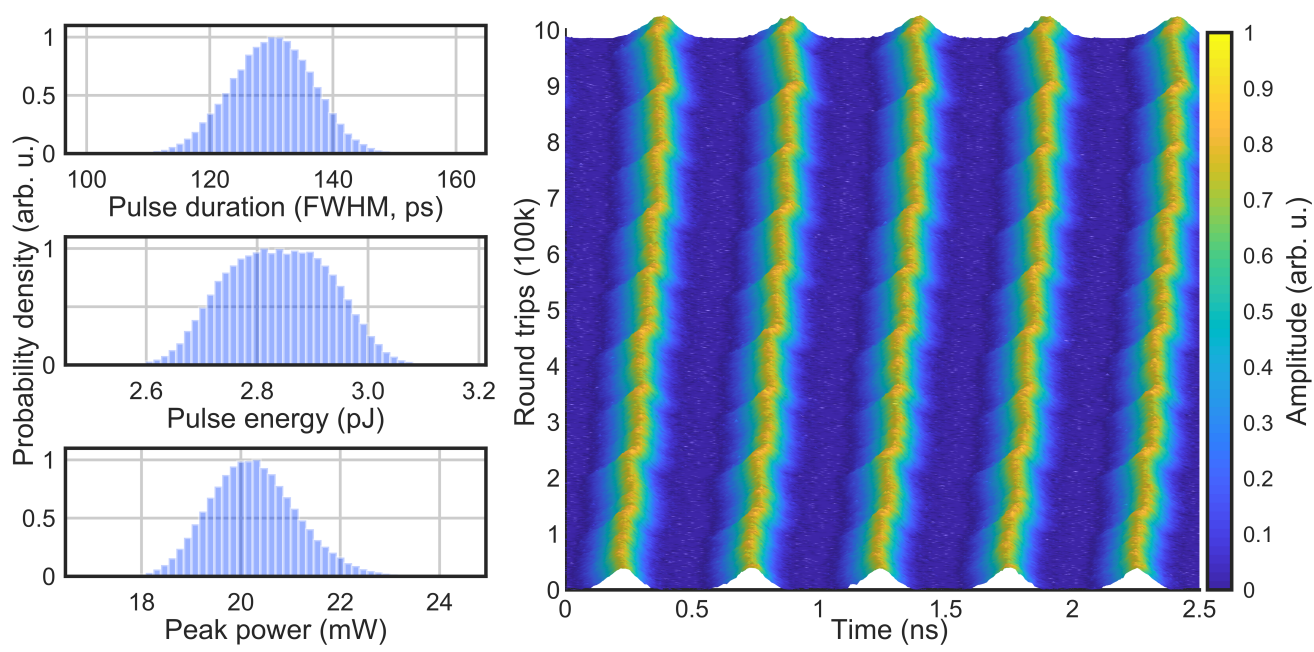

Fig. 11. Left: Distribution of pulse durations, pulse energies, and peak powers (from top to bottom) over 100,000 round trips. Right: Oscilloscope trace versus round-trip number for a time span of $500 \mu \mathrm{s}$.

Figure 11(right) shows the oscilloscope trace measured over one million round trips, corresponding to a time span of approximately $500 \mu \mathrm{s}$. The trace shows that a step-like backward shift of the pulse position occurs approximately every 100,000 round-trips, i.e. with a repetition rate of $\sim 20 \mathrm{kHz}$. This backward shift has a magnitude of approximately 30-40 ps.

\section{Appendix B: Stability analysis}

The stability analysis of the delay-differential equation model (Eq. (1) of the main text) was performed by means of the DDE-Biftool [41]. The starting point for the analysis was the trivial steady-state solution $E(t)=0, G(t)=g_{0}$ at $\varphi=0$ and the other parameters as given in the text. This solution undergoes a Hopf bifurcation at the laser threshold leading to the appearance of the steady state solution corresponding to the maximal gain mode:

$$
E(t)=A e^{i \omega t}, G(t)=G_{s}=\ln \left(\frac{\gamma^{2}+\omega^{2}}{\gamma^{2} \kappa}\right), A=\sqrt{\frac{g_{0}-G_{s}}{e^{G_{s}}-1}}
$$


,where $A$ is the constant field amplitude, $G_{s}$ is the constant gain, and $\omega$ is the constant frequency defined relatively to the gain maximum, that can be found from a transcendental equation:

$$
\omega T=\alpha \ln \left(\frac{\sqrt{\gamma^{2}+\omega^{2}}}{\gamma \sqrt{\kappa}}\right)+2 \tan ^{-1}\left(\frac{\gamma-\sqrt{\gamma^{2}+\omega^{2}}}{\omega}\right)+2 \pi M+\varphi
$$

where $M$ is the integer value corresponding to the cavity mode number. For the parameters given in the text at $\varphi=0$, the maximal gain mode is the mode $M=0$. This mode was continued in the direction of the pump parameter $g_{0}$ and then in direction of $\varphi$ for various values of $g_{0}$ to reveal the stability borders of the steady state. One of the Hopf bifurcation points leading to the stable periodic solution was used to initialize this solution, which then was continued in the direction of parameters $g_{0}$ and $\varphi$ similarly to the steady state to reveal the stability borders of the periodic solution.

The stability analysis was finalized by direct numeric integration of the model (Eq. (1) of the main text) by means of the fixed-step semi-implicit Euler method. The integration step was $0.253 \mathrm{ps}$, and the parameter $g_{0}$ was increased by $0.0014 g_{0, t h r}$ after every 30000 round trips. The parameter $\varphi$ was varied as $\varphi=a\left(g_{0} / g_{0, t h r}-1\right)$, where $a=-15 \pi$.

\section{Funding}

Engineering and Physical Sciences Research Council (EP/R024898/1); Alexander von HumboldtStiftung; H2020 Marie Skłodowska-Curie Actions (843801); Ministry of Education and Science of the Russian Federation (Passport No. 2019-1442).

\section{Acknowledgments}

This project has received funding from the European Union's Horizon 2020 research and innovation programme under the Marie Skłodowska-Curie grant agreement No 843801. N.B.C. thanks the Alexander von Humboldt Foundation for the support provided by the Feodor Lynen Research Fellowship. The work of A.V.K. and E.A.V. was supported by the Ministry of Education and Science of the Russian Federation (Passport No. 2019-1442). A.V.K. and E.A.V. acknowledge the Government of the Russian Federation (Grant 08-08).

\section{Disclosures}

The authors declare no conflict of interest.

\section{References}

1. J. McInerney, A. Mooradian, A. Lewis, A. Shchegrov, E. Strzelecka, D. Lee, J. Watson, M. Liebman, G. Carey, B. Cantos, W. Hitchens, and D. Heald, "High-power surface emitting semiconductor laser with extended vertical compound cavity," Electron. Lett. 39(6), 523-525 (2003).

2. J. G. McInerney, A. Mooradian, A. Lewis, A. V. Shchegrov, E. M. Strzelecka, D. Lee, J. P. Watson, M. K. Liebman, G. P. Carey, A. Umbrasas, C. A. Amsden, B. D. Cantos, W. R. Hitchens, D. L. Heald, V. V. Doan, and J. L. Cannon, "Novel 980-nm and 490-nm light sources using vertical cavity lasers with extended coupled cavities," Proc. SPIE 4942, 1-11 (2003).

3. T. Schwarz, M. Berens, S. Gronenborn, J. Kolb, P. Loosen, M. Miller, H. Mönch, and R. Wester, "Analysis of single-mode efficiency of electrically pumped VECSELs,” Proc. SPIE 8606, 86060H (2013).

4. H. Moench, A. Andreadaki, S. Gronenborn, J. S. Kolb, P. Loosen, M. Miller, T. Schwarz, A. van der Lee, and U. Weichmann, "High power electrically pumped VECSELs and arrays," in Proc. SPIE, J. V. Moloney, ed. (2014), 8966, p. $89660 \mathrm{H}$.

5. J.-F. Seurin, G. Xu, Q. Wang, B. Guo, R. Van Leeuwen, A. Miglo, P. Pradhan, J. D. Wynn, V. Khalfin, and C. Ghosh, "High-brightness pump sources using 2D VCSEL arrays," Proc. SPIE 7615, 76150F (2010).

6. K. Jasim, Q. Zhang, A. V. Nurmikko, A. Mooradian, G. Carey, W. Ha, and E. Ippen, "Passively modelocked vertical extended cavity surface emitting diode laser," Electron. Lett. 39(4), 373-374 (2003).

7. W. Pallmann, C. Zaugg, M. Mangold, V. Wittwer, H. Moench, S. Gronenborn, M. Miller, B. Tilma, T. Südmeyer, and U. Keller, "Gain characterization and passive modelocking of electrically pumped VECSELs," Opt. Express 20(22), 24791-24802 (2012). 
8. W. P. Pallmann, C. A. Zaugg, M. Mangold, I. Dahhan, M. Golling, B. W. Tilma, B. Witzigmann, and U. Keller, "Ultrafast Electrically Pumped VECSELs," IEEE Photonics J. 5(4), 1501207 (2013).

9. A. Alhazime, M. Butkus, C. J. Hamilton, and E. U. Rafailov, " $216 \mathrm{MHz}$ repetition rate passively mode-locked electrically-pumped VECSEL," Proc. SPIE 8966, 89660K (2014).

10. C. A. Zaugg, S. Gronenborn, H. Moench, M. Mangold, M. Miller, U. Weichmann, W. P. Pallmann, M. Golling, B. W. Tilma, and U. Keller, "Absorber and gain chip optimization to improve performance from a passively modelocked electrically pumped vertical external cavity surface emitting laser," Appl. Phys. Lett. 104(12), 121115 (2014).

11. A. G. Vladimirov and D. Turaev, "Model for passive mode locking in semiconductor lasers," Phys. Rev. A 72(3), 033808 (2005).

12. J. Mulet and S. Balle, "Mode-locking dynamics in electrically driven vertical-external-cavity surface-emitting lasers," IEEE J. Quantum Electron. 41(9), 1148-1156 (2005).

13. C. Schelte, P. Camelin, M. Marconi, A. Garnache, G. Huyet, G. Beaudoin, I. Sagnes, M. Giudici, J. Javaloyes, and S. V. Gurevich, "Third Order Dispersion in Time-Delayed Systems," Phys. Rev. Lett. 123(4), 043902 (2019).

14. M. Marconi, J. Javaloyes, S. Balle, and M. Giudici, "How Lasing Localized Structures Evolve out of Passive Mode Locking," Phys. Rev. Lett. 112(22), 223901 (2014).

15. M. Marconi, J. Javaloyes, S. Barland, S. Balle, and M. Giudici, "Vectorial dissipative solitons in vertical-cavity surface-emitting lasers with delays," Nat. Photonics 9(7), 450-455 (2015).

16. J. Javaloyes, J. Mulet, and S. Balle, "Passive Mode Locking of Lasers by Crossed-Polarization Gain Modulation," Phys. Rev. Lett. 97(16), 163902 (2006).

17. Y. F. Chen, Y. C. Lee, H. C. Liang, K. Y. Lin, K. W. Su, and K. F. Huang, "Femtosecond high-power spontaneous mode-locked operation in vertical-external cavity surface-emitting laser with gigahertz oscillation," Opt. Lett. 36(23), 4581-4583 (2011).

18. L. Kornaszewski, G. Maker, G. Malcolm, M. Butkus, E. Rafailov, and C. Hamilton, "SESAM-free mode-locked semiconductor disk laser," Laser Photonics Rev. 6(6), L20-L23 (2012).

19. A. R. Albrecht, Y. Wang, M. Ghasemkhani, D. V. Seletskiy, J. G. Cederberg, and M. Sheik-Bahae, "Exploring ultrafast negative Kerr effect for mode-locking vertical external-cavity surface-emitting lasers," Opt. Express 21(23), 28801-28808 (2013).

20. J. Moloney, I. Kilen, A. Bäumner, M. Scheller, and S. Koch, "Nonequilibrium and thermal effects in mode-locked VECSELs," Opt. Express 22(6), 6422-6427 (2014).

21. M. Gaafar, P. Richter, H. Keskin, C. Möller, M. Wichmann, W. Stolz, A. Rahimi-Iman, and M. Koch, "Self-modelocking semiconductor disk laser," Opt. Express 22(23), 28390-28399 (2014).

22. D. J. Kuizenga and A. E. Siegman, "FM and AM Mode Locking of the Homogeneous Laser-Part I: Theory," IEEE J. Quantum Electron. 6(11), 694-708 (1970).

23. D. J. Kuizenga and A. E. Siegman, "FM and AM Mode Locking of the Homogeneous Laser-Part II: Experimental Results in a Nd:YAG Laser With Internal FM Modulation," IEEE J. Quantum Electron. 6(11), 709-715 (1970).

24. L. F. Tiemeijer, P. I. Kuindersma, P. J. A. Thijs, and G. L. J. Rikken, "Passive FM Locking in InGaAsP Semiconductor Lasers," IEEE J. Quantum Electron. 25(6), 1385-1392 (1989).

25. J. Bösl, R. Bauer, H. Rauch, and U. Penning, "Modelocking of AlGaAs laser diode by intracavity AlGaAs phase-modulator," Electron. Lett. 25(13), 864-865 (1989).

26. K. A. Shore and W. M. Yee, "Theory of self-locking FM operation in semiconductor lasers," IEE Proc.-J: Optoelectron. 138(2), 91-96 (1991).

27. S. Chinn and E. Swanson, "Passive FM locking and pulse generation from 980-nm strained-quantum-well Fabry-Perot lasers," IEEE Photonics Technol. Lett. 5(9), 969-971 (1993).

28. E. Avrutin, J. Marsh, and E. Portnoi, "Monolithic and multi-GigaHertz mode-locked semiconductor lasers: Constructions, experiments, models and applications," IEE Proc.: Optoelectron. 147(4), 251-278 (2000).

29. M. Dong, S. T. Cundiff, and H. G. Winful, "Physics of frequency-modulated comb generation in quantum-well diode lasers," Phys. Rev. A 97(5), 053822 (2018).

30. J. K. Jang, M. Erkintalo, S. G. Murdoch, and S. Coen, "Ultraweak long-range interactions of solitons observed over astronomical distances," Nat. Photonics 7(8), 657-663 (2013).

31. D. V. Churkin, S. Sugavanam, N. Tarasov, S. Khorev, S. V. Smirnov, S. M. Kobtsev, and S. K. Turitsyn, "Stochasticity, periodicity and localized light structures in partially mode-locked fibre lasers," Nat. Commun. 6(1), 7004 (2015).

32. A. H. Quarterman, M. A. Tyrk, and K. G. Wilcox, "Z-scan measurements of the nonlinear refractive index of a pumped semiconductor disk laser gain medium,” Appl. Phys. Lett. 106(1), 011105 (2015).

33. A. H. Quarterman, S. Mirkhanov, C. J. C. Smyth, and K. G. Wilcox, "Measurements of nonlinear lensing in a semiconductor disk laser gain sample under optical pumping and using a resonant femtosecond probe laser," Appl. Phys. Lett. 109(12), 121113 (2016).

34. E. A. Shaw, A. H. Quarterman, A. P. Turnbull, T. Chen Sverre, C. R. Head, A. C. Tropper, and K. G. Wilcox, "Nonlinear Lensing in an Unpumped Antiresonant Semiconductor Disk Laser Gain Structure," IEEE Photonics Technol. Lett. 28(13), 1395-1398 (2016).

35. C. Kriso, S. Kress, T. Munshi, M. Grossmann, R. Bek, M. Jetter, P. Michler, W. Stolz, M. Koch, and A. Rahimi-Iman, "Microcavity-enhanced Kerr nonlinearity in a vertical-external-cavity surface-emitting laser," Opt. Express 27(9), 11914-11929 (2019).

36. J. R. Fienup, "Phase retrieval algorithms : a comparison," Appl. Opt. 21(15), 2758-2769 (1982). 


\section{Optics EXPRESS}

37. J. Peatross and A. Rundquist, "Temporal decorrelation of short laser pulses,” J. Opt. Soc. Am. B 15(1), 216-222 (1998).

38. F. Camacho, E. Avrutin, P. Cusumano, A. Saher Helmy, A. Bryce, and J. Marsh, "Improvements in mode-locked semiconductor diode lasers using monolithically integrated passive waveguides made by quantum-well intermixing," IEEE Photonics Technol. Lett. 9(9), 1208-1210 (1997).

39. M. Schell, M. Tsuchiya, and T. Kamiya, “Chirp and stability of mode-locked semiconductor lasers,” IEEE J. Quantum Electron. 32(7), 1180-1190 (1996).

40. C. Henry, "Theory of the linewidth of semiconductor lasers," IEEE J. Quantum Electron. 18(2), 259-264 (1982).

41. K. Engelborghs, T. Luzyanina, and D. Roose, "Numerical bifurcation analysis of delay differential equations using DDE-BIFTOOL," ACM Trans. Math. Softw. 28(1), 1-21 (2002).

42. A. E. Siegman, Lasers (University Science Books, 1986).

43. U. Kruger and K. Kruger, "Simultaneous measurement of the linewidth, linewidth enhancement factor $\alpha$, and FM and AM response of a semiconductor laser," J. Lightwave Technol. 13(4), 592-597 (1995).

44. H. Halbritter, F. Riemenschneider, J. Jacquet, J.-G. Provost, C. Symonds, I. Sagnes, and P. Meissner, "Chirp and linewidth enhancement factor of tunable, optically-pumped long wavelength VCSEL," Electron. Lett. 40(4), 242-243 (2004).

45. M. Marconi, J. Javaloyes, S. Balle, and M. Giudici, "Passive Mode-Locking and Tilted Waves in Broad-Area Vertical-Cavity Surface-Emitting Lasers,” IEEE J. Sel. Top. Quantum Electron. 21(1), 85-93 (2015). 